\section{A Shift Toward a Plant-Centered Diet From Young to Middle Adulthood and Subsequent Risk of Type 2 Diabetes and Weight Gain: The Coronary Artery Risk Development in Young Adults (CARDIA) Study}

Diabetes Care 2020;43:2796-2803 | https://doi.org/10.2337/dc20-1005
Yuni Choi, ${ }^{1}$ Nicole Larson, ${ }^{2}$

Daniel D. Gallaher, ${ }^{1}$ Andrew O. Odegaard, ${ }^{3}$ Jamal S. Rana, ${ }^{4,5}$ James M. Shikany, ${ }^{6}$ Lyn M. Steffen, ${ }^{2}$ and David R. Jacobs Jr. ${ }^{2}$

\section{OBJECTIVE}

To examine the associations between change in plant-centered diet quality and type 2 diabetes risk and change in body size.

\section{RESEARCH DESIGN AND METHODS}

A prospective study conducted in the U.S. enrolled adults ages 18-30 years in 19851986 (examination year [YO]) and followed them through 2015-2016. We analyzed the associations between change in plant-centered diet quality over 20 years (Y0Y20) and diabetes (Y20-30; $n=2,534)$ and change (YO-Y20 and Y20-30) in BMI, waist circumference $(W C)$, and weight $(n>2,434)$. Plant-centered diet quality was measured using the A Priori Diet Quality Score (APDQS); a higher score favors nutritionally rich plant foods. Cox regression models were used to assess diabetes risk, and linear regression models were used to examine change in body size.

\section{RESULTS}

During a mean follow-up of $9.3( \pm 1.7)$ years, 206 case subjects with incident diabetes were observed. In multivariable analysis, participants with the largest increase in APDQS over 20 years had a 48\% (95\% Cl 0.31-0.85; $\left.P_{\text {trend }}<0.001\right)$ lower risk of diabetes over the subsequent 10 years compared with participants whose score remained stable. Each 1-SD increment in APDQS over 20 years was associated with lower gains in BMI $\left(-0.39 \mathrm{~kg} / \mathrm{m}^{2} ; \mathrm{SE} 0.14 ; P=0.004\right), W C(-0.90 \mathrm{~cm} ; \mathrm{SE} 0.27$; $P<0.001)$ and weight $(-1.14 \mathrm{~kg} ; \mathrm{SE} 0.33 ; P<0.001)$ during the same period, but not with subsequent changes.

\section{CONCLUSIONS}

Young adults who increased plant-centered diet quality had a lower diabetes risk and gained less weight by middle adulthood.
${ }^{1}$ Department of Food Science and Nutrition, University of Minnesota Twin Cities, Minneapolis, MN ${ }^{2}$ Division of Epidemiology and Community Health, University of Minnesota Twin Cities, Minneapolis, $M N$

${ }^{3}$ Department of Epidemiology, School of Medicine, University of California, Irvine, Irvine, CA ${ }^{4}$ Divisions of Cardiology and Research, Kaiser Permanente Northern California, Oakland, CA ${ }^{5}$ Department of Medicine, University of California San Francisco, San Francisco, CA

${ }^{6}$ Division of Preventive Medicine, School of Medicine, University of Alabama at Birmingham, Birmingham, AL

Corresponding author: David R. Jacobs Jr., jacob004 @umn.edu

Received 1 May 2020 and accepted 27 July 2020

This article contains supplementary material online at https://doi.org/10.2337/figshare.12733598.

This article is featured in a podcast available at https://www.diabetesjournals.org/content/ diabetes-core-update-podcasts.

(C) 2020 by the American Diabetes Association. Readers may use this article as long as the work is properly cited, the use is educational and not for profit, and the work is not altered. More information is available at https://www.diabetesjournals .org/content/license. 
Increased incidence of type 2 diabetes and obesity have been major public health problems in the U.S. Weight gain in young adulthood may lead to an earlier onset of type 2 diabetes and greater risk of heart disease and all-cause mortality $(1,2)$. Recently, the EAT-Lancet Commission pointed to the benefits that a plantcentered diet has on health and the environment (3). Previous studies reported that a plant-centered diet was associated with a lower risk of type 2 diabetes and weight gain $(4,5)$. However, only a few previous studies focused on change in diet quality over time (started following participants when they were middle-aged) (6-8). These studies showed that diets that generally emphasized plant foods were associated with a lower subsequent risk of type 2 diabetes and concurrent weight gain (6-8). Given the importance of the transition period from young to middle adulthood for preventing chronic disease, young adults may benefit from increasing plant-centered diet quality, as this may help to prevent type 2 diabetes and weight gain.

In the current study, diet quality was measured using the A Priori Diet Quality Score (APDQS); high index scores were characterized by higher consumption of nutritionally rich plant foods with limited consumption of meats and less healthful plant foods. Although no food is forbidden, a high score requires a variety of food choices, which in turn enhances flexibility. The APDQS embodies many principles of the 2015-2020 Dietary Guidelines for Americans and was found to predict risk of myocardial infarction, mortality, and other clinical outcomes (9-12). A previous study found a relationship between cumulative APDQS and type 2 diabetes over 30 years (11). The current study modeled both baseline and long-term change in diet quality from young to middle adulthood as exposures and incidence of type 2 diabetes and change in body size as outcomes. We hypothesized that baseline and 20-year change in plant-centered diet quality predict subsequent 10 -year risk of type 2 diabetes, as well as concurrent and subsequent change in BMI, waist circumference (WC), and weight.

\section{RESEARCH DESIGN AND METHODS}

Study Design and Participants

Coronary Artery Risk Development in Young Adults (CARDIA) is a communitybased, prospective cohort study of 5,115
Black and White men and women aged 18-30 years (1985-1986, examination year [YO]) who were recruited from four U.S. cities (13). Study recruitment balanced the age, sex, race, and education of the participants within each study center. Nine examinations were conducted, with high retention over 30 years of follow-up among survivors $(71 \%$ at Y30, 20152016). All participants provided written informed consent, and the research protocols were approved by institutional review boards at each CARDIA site.

For the 3,549 participants who attended CARDIA at Y20, exclusion criteria were: did not complete the dietary history questionnaire at Y0 and Y20 ( $n=$ 409); had an implausible energy intake ( $<800$ or $>8,000 \mathrm{kcal} /$ day for men; $<600$ or $>6,000 \mathrm{kcal} /$ day for women; $n=514$ ); or did not provide information regarding smoking status at $Y 0$ or physical activity at $\mathrm{YO}$ or $\mathrm{Y} 20(n=43)$. For the analysis of diabetes, participants were excluded who had diabetes in Y0-Y20 ( $n=332$ ) or were not examined at $Y 25$ or Y30 (no assessment of incident type 2 diabetes, $n=238$ ). For body size change, participants with missing BMI $(n=628)$, WC $(n=631)$, or weight $(n=622)$ at Y0, Y20, or Y30 or who reported bariatric surgery $(n=81)$ were excluded. The final sample number for diabetes analysis was 2,534 participants and 2,436 for BMI, 2,434 for WC, and 2,439 for weight. Comparison of the $\mathrm{YO}$ characteristics of excluded versus included participants showed that those excluded were more likely to report lower educational attainment, identify as Black race, and smoke cigarettes and to have lower APDQS and higher BMI and diabetes (Supplementary Table 1). Nevertheless, all population subgroups were well represented in those included.

\section{Assessment of Plant-Centered Diet Quality}

Diet was assessed using the intervieweradministered, validated CARDIA diet history questionnaire at $Y 0$ and was updated at Y7 (1992-1993) and Y20 (2005-2006) $(14,15)$. Interviewers asked 100 openended questions about food and beverage consumption over the past month. The frequency of consumption, unit or serving size, and preparation method were recorded. The number of food items that were collected was 950 at $Y 0,1,388$ at $Y 7$, and 4,598 at $\mathrm{Y} 20$.
Plant-centered diet quality was measured by the APDQS, which is a hypothesisdriven index of 46 food groups. The APDQS is based on food groups classified as beneficial $(n=20)$, adverse $(n=13)$, and neutral ( $n=13$ ) according to their hypothesized relationship with cardiovascular disease. The scoring system of APDQS is based on quintile rankings of each of the 46 food groups (for food groups with large zero servings per day, a zero and quartiles above zero were used). The study-specific cut points derived at YO were applied to follow-up data, which allowed change in participants' dietary patterns to be tracked. Beneficially rated food groups were assigned points ranging from 0 (lowest quintile) to 4 (highest quintile), adversely rated food groups were assigned points ranging from 0 (highest quintile) to 4 (lowest quintile), and zero points were assigned to neutral food groups. The APDQS corresponded to the sum of the 46 component scores and had possible scores ranging between 0 and 132 , with a range of 35-95 for the data collected in this study. For the current analysis, 20-year change in diet quality was subtracted from the value at $Y O$ from the value at $\mathrm{Y} 20$.

Supplementary Table 2 describes the feature of plant-centeredness in APDQS by comparing the mean intake of the 46 food groups between the extreme groups ("high initial and increased," defined as at or above the median YO APDQS and quintile 5 of 20-year change vs. "Iow initial and decreased," defined as below the median YO APDQS and quintile 1 of 20-year change). Supplementary Table 3 describes how the "high initial and increased" group made changes to their diet to obtain the highest increasing diet score over time. Notably, this group greatly increased their consumption of beneficially rated plant foods compared with the "low initial and decreased" group over a 20-year period. Subfood groups constituting the 46 food groups are shown in Supplementary Table 4.

\section{Ascertainment of Type 2 Diabetes}

Type 2 diabetes was diagnosed if fasting glucose concentration was $\geq 126$ $\mathrm{mg} / \mathrm{dL}$, the 2 -h postchallenge glucose concentration was $\geq 200 \mathrm{mg} / \mathrm{dL}$ (measured at Y10, Y20, and Y25), glycated hemoglobin $\left(\mathrm{HbA}_{1 \mathrm{c}}\right)$ was $\geq 6.5 \%$ (48 mmol/ $\mathrm{mol})$ at $\mathrm{Y} 20$ and $\mathrm{Y} 25$, and/or use of antidiabetic medications was self-reported 
(per medication bottle brought to clinic). Incident type 2 diabetes was diagnosed if this condition was first satisfied at $\mathrm{Y} 25$ or Y30.

\section{Anthropometric Measurements}

With participants barefoot and wearing light clothing, height was measured to the nearest $0.5 \mathrm{~cm}$ with a vertical ruler, weight was measured to the nearest $0.2 \mathrm{~kg}$ with a calibrated balance beam scale, and WC was measured to the nearest $0.5 \mathrm{~cm}$ with a tape in duplicate. BMI was calculated as weight divided by height squared $\left(\mathrm{kg} / \mathrm{m}^{2}\right)$.

\section{Assessment of Covariates}

Standardized questionnaires were used to collect demographics, parental history of diabetes, smoking, and physical activity at all CARDIA exams. Physical activity level was assessed by a trained interviewer using the CARDIA physical activity history questionnaire (16). Participants were asked to report the frequency of 13 exercise activities over the previous year. The total score was the product of intensity and frequency.

\section{Statistical Analysis}

The focus of this study was change in diet quality from young to middle adulthood (over 20 years, Y0-Y20). Proportional hazards regression was used to estimate hazard ratios (HRs) and $95 \% \mathrm{Cls}$. Of main interest was the joint association of the continuous YO APDQS and 20-year change in APDQS with type 2 diabetes $\left(P_{\text {interaction }}=0.94\right)$. Model 1 was adjusted for age, sex, race (White or Black), and energy intake (YO and 20-year change). Model 2 was further adjusted for parental history of diabetes, physical activity level (tertiles; YO and 20-year change), YO smoking (never, former, and current), education, and BMI (YO and 20-year change). For the smoking variable, information at YO was used because the time-varying smoking status variable did not contribute to predictions. We evaluated the extent to which the relationship between 20-year change in APDQS and diabetes risk was mediated by concurrent change in BMI, WC, and weight. Mediation analysis estimated the degree of the mediation effect by comparing the models with and without the mediating variables and quantified the difference in estimates between these two models: $1-\left(\beta_{\text {mediator model }} / \beta_{\text {base model }}\right) *$ 100 (17). Goodness of linear fit of the continuous models in the joint association with $\mathrm{YO}$ and 20-year change in APDQS was assessed in two ways. First, to visually assess the shape of association of YO APDQS and 20-year change in APDQS (both as continuous) with diabetes, restricted cubic splines with four knots were computed. Nonlinearity was tested using likelihood ratio tests by comparing two models: 1) model with the linear term and 2) the model with the linear and cubic spline terms in model 2 (18). Second, the combined effect of YO (median) and 20-year change (quintiles) in APDQS on diabetes incidence was estimated. A separate analysis was conducted that examined whether Y20 APDQS predicted diabetes over 10 years. We tested for interactions between 20-year change in APDQS and age, race, sex, and education.

Next, we evaluated the associations of YO APDQS and 20-year APDQS change with concurrent (YO-Y20) and subsequent (Y20-Y30) change in $\mathrm{BMI}, \mathrm{WC}$, and weight. The multivariable models were adjusted for the same covariates as the diabetes analysis, omitting parental history of diabetes. YO BMI, WC, or weight was also adjusted in the model. Additional analyses were performed that examined whether YO APDQS predicted change in BMI, WC, or weight over 30 years and whether Y20 APDQS predicted the same outcomes over 10 years in which the same covariates were adjusted but only Y0 or Y20 variables were included in the models.

Sensitivity analyses examined whether short-term change (7-year change) in APDQS was consistently associated with diabetes risk or change in body size. The same modeling strategies were applied to this analysis as were applied to the analysis of 20-year change. In addition, individual food group analyses were conducted by fitting two main distinctive APDQS food groups regarding nutritional and health value, beneficially rated plant food groups (fruit, avocado, beans/legumes, green vegetables, yellow vegetables, tomatoes, other vegetables, nuts and seeds, soy products, whole grain, and vegetable oil), and adversely rated animal food groups (high-fat meats, processed meats, organ meats, fried fish/poultry, and sauces), with adjustment for the rest of the APDQS food groups in separate multivariable analyses. SAS software version 9.4 (SAS Institute Inc., Cary, NC) was used in all analyses. Statistical tests were two-tailed, with significance at $P<0.05$.

\section{RESULTS}

\section{Characteristics}

We identified 206 new case subjects with type 2 diabetes during mean follow-up of 9.3 ( \pm 1.7 ) years after Y20 APDQS was measured. Twenty-year changes in APDQS were inversely related to $Y 0$ APDQS, energy intake, and current smoking within both categories of below the median and at or above the median YO APDQS. Twenty-year changes in APDQS were positively related to the proportion of females and Whites and 20-year change in alcohol intake (Table 1). A greater increase in APDQS over 20 years was associated with a smaller decrease in the level of physical activity. Twenty-year changes in APDQS were negatively associated with 20-year change in WC, BMI, and weight only for at or above the median YO APDQS.

Prediction of Incident Type 2 Diabetes Y20 APDQS predicted type 2 diabetes risk over 10 years (HR 0.68 [95\% Cl 0.57-0.80] per 1-SD increment in Y20 APDQS) (model 2 in Table 2). In joint predictor models, both YO and 20-year APDQS change predicted diabetes. Fully adjusted analyses showed a $37 \%(95 \% \mathrm{Cl} 0.51-0.78)$ reduction in diabetes risk per 1-SD increment in YO APDQS and a $29 \%(95 \% \mathrm{Cl} 0.59$ $0.86)$ decrease in diabetes risk per 1-SD increment in change in APDQS over 20 years. These patterns were reiterated in spline analysis (Supplementary Fig. 1). Mediation effect analyses showed that $31.5 \%$ (95\% Cl 12.3-60.0\%; $P<0.001$ ) of the association between 20-year change in APDQS and diabetes risk was explained by concurrent change in BMI, WC, and weight.

Added specificity is shown in Table 3, in which individuals who started with a higher plant-centered diet quality in young adulthood and increased the most over 20 years (at or above median YO APDQS and quintile 5 of 20-year change) had a $95 \%(95 \% \mathrm{Cl} 0.01-0.44)$ lower subsequent 10 -year risk of diabetes compared with those who had a lower diet quality in young adulthood and remained stable (<median YO APDQS and quintile 2 of 20 -year change) over 20 years. However, these results should be cautiously interpreted due to the small sample size of this category. The cumulative incidence of diabetes 
Table 1-Characteristics of the participants according to 20 -year change* in APDQSt, stratified by YO APDQS ( $n=2,534$ )

\begin{tabular}{|c|c|c|c|c|c|c|}
\hline \multirow[b]{3}{*}{ Characteristics } & \multicolumn{3}{|c|}{ Below the median YO APDQS } & \multicolumn{3}{|c|}{ At or above the median YO APDQS } \\
\hline & \multicolumn{3}{|c|}{ 20-year change in APDQS } & \multicolumn{3}{|c|}{ 20-year change in APDQS } \\
\hline & $\begin{array}{l}\text { Quintile } 1 \\
(n=126)\end{array}$ & $\begin{array}{l}\text { Quintile } 3 \\
(n=252)\end{array}$ & $\begin{array}{l}\text { Quintile } 5 \\
(n=377)\end{array}$ & $\begin{array}{l}\text { Quintile } 1 \\
(n=363)\end{array}$ & $\begin{array}{l}\text { Quintile } 3 \\
(n=288) \\
\end{array}$ & $\begin{array}{l}\text { Quintile } 5 \\
(n=112)\end{array}$ \\
\hline \multicolumn{7}{|l|}{ APDQS } \\
\hline YO & $57.4 \pm 4.6$ & $53.9 \pm 6.3$ & $51.1 \pm 7.4$ & $78.7 \pm 9.3$ & $73.7 \pm 7.3$ & $69.7 \pm 5.3$ \\
\hline 20-year change* & $-7.9 \pm 3.9$ & $6.6 \pm 1.8$ & $23.7 \pm 6.1$ & $-9.6 \pm 5.7$ & $6.5 \pm 1.7$ & $21.3 \pm 4$ \\
\hline YO age, years & $24.6 \pm 3.5$ & $24.6 \pm 3.7$ & $23.7 \pm 3.8$ & $25.9 \pm 3.3$ & $25.9 \pm 3.1$ & $25.6 \pm 3.2$ \\
\hline Female, $n(\%)$ & $55(43.7)$ & $146(57.9)$ & $215(57)$ & $215(59.2)$ & $166(57.6)$ & $74(66.1)$ \\
\hline White race, $n(\%)$ & 44 (34.9) & $80(31.8)$ & $141(37.4)$ & $262(72.2)$ & $241(83.7)$ & $93(83)$ \\
\hline Highest grade of education, years & $14.7 \pm 2.6$ & $15 \pm 2.6$ & $15.7 \pm 2.6$ & $16.5 \pm 2.4$ & $16.8 \pm 2.4$ & $17 \pm 2.3$ \\
\hline Parental history of diabetes, $n$ (\%) & $36(28.6)$ & $80(31.8)$ & $106(28.1)$ & $82(22.6)$ & $53(18.4)$ & $28(25)$ \\
\hline \multicolumn{7}{|l|}{ Physical activity, EU¥ } \\
\hline YO & $401 \pm 331$ & $344 \pm 269$ & $382 \pm 278$ & $483 \pm 327$ & $486 \pm 285$ & $451 \pm 248$ \\
\hline 20-year change* & $-148 \pm 305$ & $-81.3 \pm 248$ & $-20.6 \pm 283$ & $-151 \pm 310$ & $-71.8 \pm 285$ & $16.2 \pm 274$ \\
\hline YO current smoker, $n(\%)$ & $43(34.1)$ & $71(28.2)$ & $79(21)$ & $86(23.7)$ & $63(21.9)$ & $19(17)$ \\
\hline \multicolumn{7}{|l|}{ Alcohol intake, drinks/day } \\
\hline YO & $1.08 \pm 1.68$ & $0.64 \pm 1.28$ & $0.46 \pm 0.84$ & $1.1 \pm 1.43$ & $0.94 \pm 1.12$ & $1.04 \pm 1.44$ \\
\hline 20-year change* & $-0.67 \pm 1.72$ & $-0.05 \pm 1.65$ & $0.31 \pm 1.17$ & $-0.26 \pm 1.49$ & $0.1 \pm 1.41$ & $0.23 \pm 1.45$ \\
\hline \multicolumn{7}{|l|}{ Total energy intake, kcal/day } \\
\hline YO & $3,253 \pm 1,573$ & $2,892 \pm 1,425$ & $2,873 \pm 1,283$ & $2,658 \pm 1,146$ & $2,507 \pm 1,108$ & $2,466 \pm 1,061$ \\
\hline 20-year change* & $-488 \pm 1,473$ & $-634 \pm 1,334$ & $-578 \pm 1,324$ & $-325 \pm 1,126$ & $-251 \pm 1,014$ & $-141 \pm 1,104$ \\
\hline $\begin{array}{l}\text { Case subjects with incident type } 2 \\
\text { diabetes (Y20-Y30), } n(\%)\end{array}$ & $19(15.1)$ & $31(12.3)$ & $28(7.4)$ & $26(7.2)$ & $16(5.6)$ & $2(1.8)$ \\
\hline \multicolumn{7}{|l|}{ BMI, $\mathrm{kg} / \mathrm{m}^{2}$} \\
\hline YO & $25.2 \pm 5.6$ & $24.6 \pm 5$ & $23.9 \pm 4.4$ & $23.9 \pm 3.9$ & $23.3 \pm 3.8$ & $23.1 \pm 4.5$ \\
\hline 20-year change* & $5.3 \pm 4$ & $5.7 \pm 6.4$ & $5.3 \pm 4.3$ & $4.6 \pm 4.5$ & $3.8 \pm 4$ & $4.1 \pm 11$ \\
\hline \multicolumn{7}{|l|}{$W C, \mathrm{~cm}$} \\
\hline YO & $80.1 \pm 11.9$ & $77.8 \pm 10.7$ & $76 \pm 9.8$ & $76.1 \pm 9.7$ & $75.5 \pm 9.4$ & $74.5 \pm 9.9$ \\
\hline 20-year change* & $14.8 \pm 8.6$ & $15.6 \pm 12$ & $14.4 \pm 9.3$ & $13.4 \pm 10.3$ & $11.5 \pm 9.7$ & $9.4 \pm 7.8$ \\
\hline \multicolumn{7}{|l|}{ Weight, kg } \\
\hline YO & $74.1 \pm 17.1$ & $71.0 \pm 15.1$ & $69.0 \pm 14.2$ & $69.0 \pm 14.0$ & $68.4 \pm 13.6$ & $66.4 \pm 13.2$ \\
\hline 20-year change* & $15.8 \pm 11.2$ & $16.5 \pm 15.1$ & $15.7 \pm 11.8$ & $13.7 \pm 13.1$ & $11.3 \pm 11.5$ & $9.4 \pm 10.2$ \\
\hline
\end{tabular}

Data are mean \pm SD unless otherwise indicated. Median cut point of YO APDQS was $64 .{ }^{*}$ The 20 -year change was calculated by subtracting the Y0 values from the follow-up values at $Y 20$. †Total score sums the 46 components (possible scores $0-132$, with a range of $35-95$ in these data), with higher scores representing a nutritionally rich, plant-centered diet. A one-point increment represents a one-category shift in the presumed favorable direction. $¥ E U$ is exercise units, a physical activity score derived from the CARDIA physical activity history.

corresponding to these two categories was $12.2 \%$ and $1.8 \%$, respectively, which is an $\sim 5.8$-fold difference in the incidence rate. Similar, but weaker, inverse association was observed for 7-year change in APDQS in relation to risk of diabetes in the subsequent 23-year period (Supplementary Tables 5 and 6). We found no difference in the association by stratified variables, including age, race, sex, and education $\left(P_{\text {interaction }}>0.05\right.$ for each). In analyses of individual food groups, beneficially rated plant foods were inversely associated with diabetes (HR 0.45 [95\% Cl 0.26-0.78] for $\mathrm{YO}$ and HR 0.84 [95\% Cl 0.60-1.17] for 20-year change), whereas adversely rated animal foods were positively associated with diabetes (HR 2.26 [95\% Cl 1.18-4.30] for $\mathrm{YO}$ and $\mathrm{HR} 2.25$ [95\% Cl 1.23-4.13] for 20-year change).

\section{Prediction of Change in Measures of Body Size}

The mean increase in $\mathrm{BMI}, \mathrm{WC}$, and weight over 30 years was $6.1 \mathrm{~kg} / \mathrm{m}^{2}$ ( \pm 5.2 ),

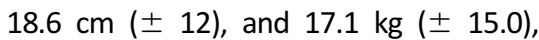
respectively. YO APDQS predicted a smaller increase in body size over 30 years; the adjusted $\beta \pm$ SE per 1 SD was $-0.64 \pm$ $0.13 \mathrm{~kg} / \mathrm{m}^{2}(P<0.001)$ for BMl, $-1.58 \pm$ $0.3 \mathrm{~cm}$ for WC $(P<0.001)$, and -1.62 $\pm 0.36 \mathrm{~kg}$ for weight $(P<0.001)$.

In models that simultaneously adjusted for YO APDQS and 20-year change in APDQS, 20-year change in APDQS was associated with a smaller concurrent increase in BMI $\left(-0.39 \mathrm{~kg} / \mathrm{m}^{2}\right.$; SE 0.14; $P=0.004)$, WC $(-0.90 \mathrm{~cm} ;$ SE $0.27 ; P<$ $0.001)$, and weight $(-1.14 \mathrm{~kg}$; SE 0.33; $P<0.001)$, and YO APDQS more strongly predicted change in $\mathrm{BMI}\left(-0.65 \mathrm{~kg} / \mathrm{m}^{2} ; \mathrm{SE}\right.$ 0.14 ; $P<0.001)$, WC $(-1.35 \mathrm{~cm}$; SE 0.29;
$P<0.001)$, and weight $(-1.65 \mathrm{~kg}, \mathrm{SE} 0.36$; $P<0.001)$ than 20-year change in APDQS (Table 4). YO APDQS, but not 20-year change in APDQS, was associated with a lower increase in BMI $\left(-0.37 \mathrm{~kg} / \mathrm{m}^{2}\right.$; SE $0.11 ; P<0.001)$, WC $(-0.77 \mathrm{~cm}$; SE $0.22 ; P<0.001)$, and weight $(-0.61 \mathrm{~kg}$; SE 0.25; $P=0.014$ ) in the subsequent period. The results were similar for 7-year change in APDQS (Supplementary Table 7).

\section{CONCLUSIONS}

The current study showed that increased plant-centered diet quality over 20 years, beginning in young adulthood, was associated with a lower risk of type 2 diabetes by middle adulthood. The current study extends a previous analysis of the same database, which showed that the cumulative average APDQS was associated with the risk of type 2 diabetes 


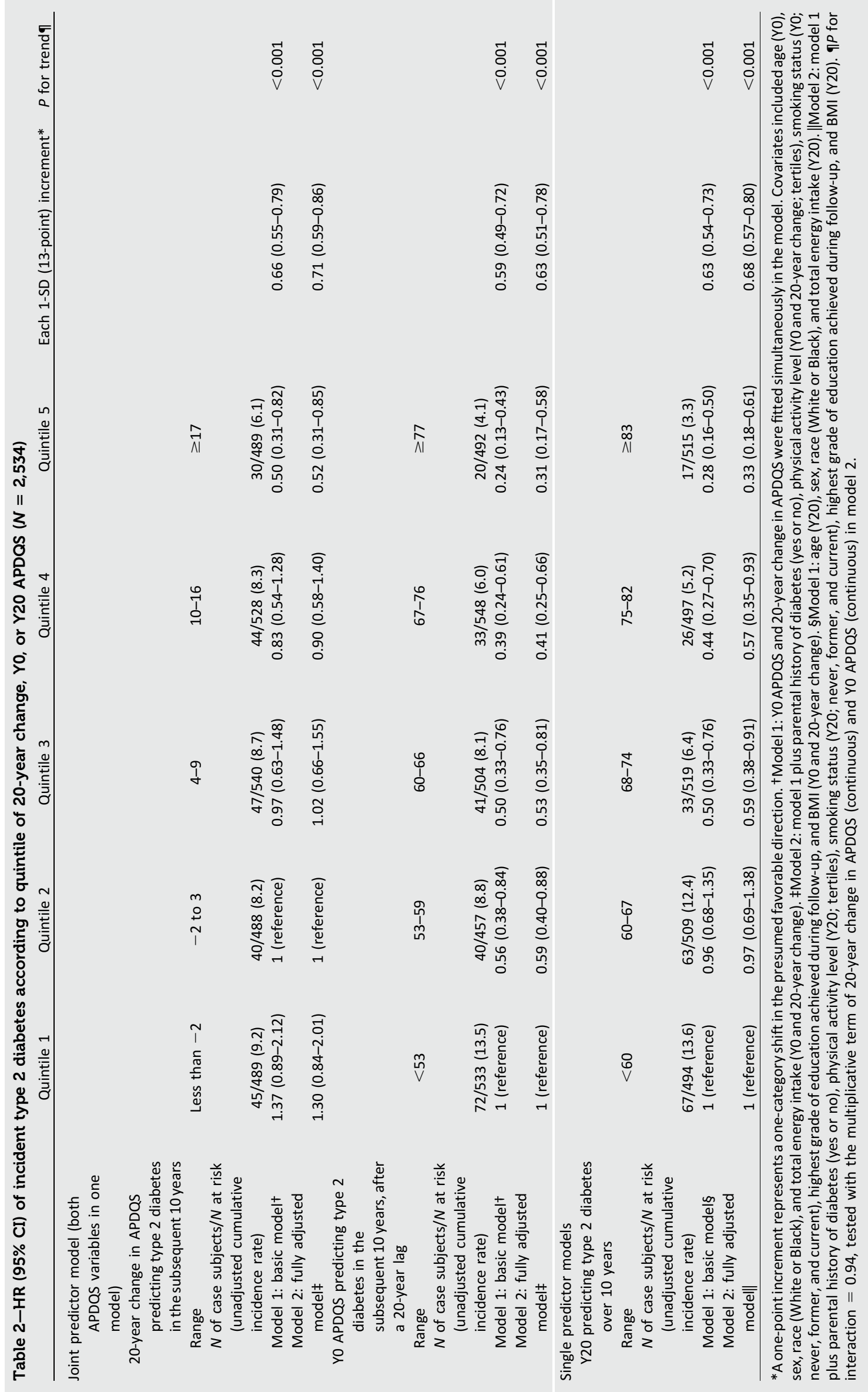


Table 3-Subsequent 10-year HR (95\% CI) of incident type 2 diabetes according to joint classification of YO APDQS and 20 -year change in APDQS $(n=2,534)$

20-year change in APDQS

\begin{tabular}{|c|c|c|c|c|c|}
\hline & & & & & \\
\hline & Quintile 1 & Quintile 2 & Quintile 3 & Quintile 4 & Quintile 5 \\
\hline \multicolumn{6}{|l|}{ Below the median of YO APDQS $(n=1,272)$} \\
\hline Range & Less than -2 & -2 to 3 & $4-9$ & $10-16$ & $\geq 17$ \\
\hline \multicolumn{6}{|l|}{$N$ of case subjects $/ N$ at risk } \\
\hline (unadjusted cumulative incidence rate) & $19 / 126(15.1)$ & 23/189 (12.2) & $31 / 252(12.3)$ & $35 / 328(10.7)$ & 28/377 (7.4) \\
\hline Unadjusted & $1.27(0.69-2.33)$ & 1 (reference) & $1.03(0.60-1.76)$ & $0.87(0.52-1.47)$ & $0.60(0.34-1.04)$ \\
\hline Model 1: basic model* & $1.21(0.66-2.23)$ & 1 (reference) & $1.00(0.59-1.72)$ & $0.93(0.55-1.57)$ & $0.62(0.36-1.08)$ \\
\hline Model 2: fully adjusted model ${ }^{\dagger}$ & $1.22(0.66-2.24)$ & 1 (reference) & $1.03(0.60-1.77)$ & $1.00(0.59-1.71)$ & $0.72(0.41-1.27)$ \\
\hline \multicolumn{6}{|c|}{ At or above the median of YO APDQS $(n=1,262)$} \\
\hline Range & Less than -2 & -2 to 3 & $4-9$ & $10-16$ & $\geq 17$ \\
\hline \multicolumn{6}{|l|}{$N$ of case subjects $/ N$ at risk } \\
\hline (unadjusted cumulative incide & $26 / 363(7.2)$ & $17 / 299(5.7)$ & $16 / 288(5.6)$ & $9 / 200(4.5)$ & $2 / 112(1.8)$ \\
\hline Unadjusted & $0.58(0.33-1.02)$ & $0.45(0.24-0.85)$ & $0.44(0.23-0.83)$ & $0.37(0.17-0.79)$ & $0.14(0.03-0.60)$ \\
\hline Model 1: basic model* & $0.70(0.39-1.24)$ & $0.57(0.30-1.07)$ & $0.58(0.30-1.12)$ & $0.47(0.22-1.04)$ & $0.19(0.04-0.80)$ \\
\hline Model 2: fully adjusted model ${ }^{\dagger}$ & $0.77(0.43-1.37)$ & $0.68(0.36-1.30)$ & $0.79(0.40-1.53)$ & $0.69(0.31-1.54)$ & $0.05(0.01-0.44)$ \\
\hline
\end{tabular}

Median cut point of YO APDQS was 64. *Model 1: age (YO), sex, race (White or Black), and total energy intake (YO and 20-year change). + Model 2: model 1 plus parental history of diabetes (yes or no), physical activity level ( $\mathrm{YO}$ and 20-year change; tertiles), smoking status ( $\mathrm{YO}$; never, former, and current), highest grade of education achieved during follow-up, and BMI (YO and 20-year change).

over a 30-year period, with an HR of 0.55 that compared quartile 4 versus quartile 1 (11). In the current study, participants with the greatest increase in plant-centered diet score over 20 years had a $48 \%$ lower risk of type 2 diabetes over the next 10 years as compared with those with a stable score. Furthermore, individuals who had a higher diet quality in young adulthood and increased over time showed a greater decrease in risk for diabetes. Increased diet quality over 20 years was associated with lower gains in BMI, WC, and weight.

The global data showed that increased consumption of plant-centered diets predicted a $23.6 \%$ reduction in deaths (11.6 million/year) and an $80 \%$ reduction in greenhouse gas emissions (3). Our findings are consistent with a metaanalysis of nine prospective studies that showed an inverse association between
Table 4-Adjusted mean change* in BMI and WC per 1-SD increment in 20-year change, Y0, or Y20 APDQS

\begin{tabular}{|c|c|c|c|c|c|c|}
\hline \multirow[b]{2}{*}{ Each 1-SD (13-point) increment } & \multicolumn{3}{|c|}{$\begin{array}{l}\text { Concurrent 20-year change } \\
\text { (YO-Y20) }\end{array}$} & \multicolumn{3}{|c|}{$\begin{array}{l}\text { Subsequent 10-year change } \\
\text { (Y20-Y30) }\end{array}$} \\
\hline & $\beta$ & SE & $P$ value & $\beta$ & SE & $P$ value \\
\hline \multicolumn{7}{|l|}{$\mathrm{BMI}, \mathrm{kg} / \mathrm{m}^{2}(n=2,436)$} \\
\hline 20-year change $(\mathrm{YO}-20)^{\dagger}$ & -0.39 & 0.14 & 0.004 & -0.17 & 0.10 & 0.09 \\
\hline YO† & -0.65 & 0.14 & $<0.001$ & -0.37 & 0.11 & $<0.001$ \\
\hline Y20‡ & - & - & - & -0.24 & 0.09 & 0.008 \\
\hline \multicolumn{7}{|l|}{ WC, $\mathrm{cm}(n=2,434)$} \\
\hline 20-year change $(\mathrm{YO}-20)^{\dagger}$ & -0.90 & 0.27 & $<0.001$ & -0.10 & 0.21 & 0.64 \\
\hline Yo+ & -1.35 & 0.29 & $<0.001$ & -0.77 & 0.22 & $<0.001$ \\
\hline Y20‡ & - & - & - & -0.31 & 0.19 & 0.10 \\
\hline \multicolumn{7}{|l|}{ Weight, $\mathrm{kg}(n=2,439)$} \\
\hline 20 -year change $(\mathrm{YO}-20)^{\dagger}$ & -1.14 & 0.33 & $<0.001$ & -0.05 & 0.23 & 0.84 \\
\hline YO+ & -1.65 & 0.36 & $<0.001$ & -0.61 & 0.25 & 0.014 \\
\hline Y20‡ & - & - & - & -0.20 & 0.21 & 0.32 \\
\hline
\end{tabular}

*Multivariable linear models are reported. Model was adjusted for current age, sex, race (White or Black), total energy intake (YO and 20-year change), smoking status (YO; never, former, and current), physical activity level ( $\mathrm{YO}$ and 20-year change; tertiles), and highest grade of education achieved during follow-up. †oint predictor models. Each model included YO APDQS and 20-year change in APDQS. Depending on outcome variables, the following variables were also included in the model: $Y 0 \mathrm{BMI}$ for concurrent 20-year change in BMI, Y20 BMI for subsequent 10-year change in $\mathrm{BMI}, \mathrm{YO}$ WC for concurrent 20-year change in WC, and Y2O WC for subsequent 10-year change in WC, YO weight for concurrent 20-year change in weight, and Y20 weight for subsequent 10-year change in weight. $¥$ Single predictor models. the baseline level of a healthy plantcentered diet and risk of type 2 diabetes (4). Two prospective studies investigated the relationship between change in diet quality (measured using dietary guidelinebased indices) and the subsequent diabetes risk in middle-aged adults $(6,19)$. One of these studies found that 4-year increase in diet quality (assessed using the Alternate Healthy Eating Index-2010 [AHEI-2010] score) was associated with an $11 \%$ lower subsequent 4 -year risk of diabetes for every $10 \%$ increment in the score (6). Similar to our findings, this study demonstrated that the association between change in diet quality and subsequent risk of diabetes was modestly explained by change in weight (32\%). In contrast, the other study found no association between change in diet quality (assessed using the Health Eating Index2015 [HEI-2015] and the AHEI-2010) over 6 years and risk of type 2 diabetes over the following 18 years (19). Few data exist regarding long-term change in plantcentered diet quality and subsequent risk of type 2 diabetes in young adults. Our data support dietary patterns that emphasize plant foods, but also allow for some lean meats and low-fat dairy, as suggested by the 2015-2020 Dietary Guidelines for Americans (9). The APDQS strongly correlated with HEI-2015 (agesex-adjusted correlation coefficient was 0.73 in CARDIA participants) and predicted clinical outcomes (10-12). Based on our data and previous studies of individual foods, we propose making 
nutritionally rich plant foods the central feature of a diet. Nevertheless, modest amounts of dairy, fish/seafood, poultry, and eggs may be flexibly incorporated into the diet while achieving reduced diabetes risk. A meta-analysis of prospective studies reported that total dairy (whole and low fat) was inversely associated with incident type 2 diabetes (20); particularly, risk of diabetes decreased by $14 \%$ with increased yogurt intake, up to $80 \mathrm{~g} /$ day. There was no association between egg consumption and type 2 diabetes risk comparing the highest versus lowest intake in meta-analysis, but modestly increased risk was observed for more than three eggs per week only among U.S. populations (21). Another meta-analysis study shows no evidence of an increased risk of type 2 diabetes with intake of fish, shellfish, and poultry $(22,23)$. Additional studies are needed to clarify to what extent individual animal products may partially affect the risk of diabetes.

Prior observational studies demonstrated that adherence to plant-centered dietary patterns at baseline was associated with lower weight gain $(5,24)$. Likewise, a meta-analysis of 12 clinical trials found that vegetarian diets were associated with weight reduction in the general population (25). However, the duration of the studies in the meta-analysis were short (median 18 weeks), and the association was weaker for subjects with longer follow-up ( $\geq 1$ year) than for subjects with shorter follow-up ( $<1$ year), which complicates the interpretation of the long-term effects. Pooled data from three U.S. prospective cohort studies showed that increased diet quality at each 4-year interval over 20 years (assessed using the AHEI-2010, the Alternate Mediterranean Diet, and the Dietary Approaches to Stop Hypertension) was associated with a lower concurrent weight gain $(-0.47 \mathrm{~kg},-0.23 \mathrm{~kg}$, and $-0.42 \mathrm{~kg}$, respectively, for each 1-SD increment in these indices) (7). Later, the same U.S. prospective cohort studies described the relationship between change in plantbased diet indices (PDI) over 4 years and concurrent weight change during the same period (8). The study found that each 1-SD increment in the healthful PDI (positive scores were assigned to nutritious plant foods and negative scores to animal foods) was associated with 0.68 $\mathrm{kg}$ less weight gain, whereas each 1-SD increment in the unhealthful PDI (positive score assigned to highly processed plant foods and negative scores to animal foods) was associated with $0.36 \mathrm{~kg}$ more weight gain. However, these studies relied on self-reported weight measurements as opposed to the preferred objective measurements and may bias the relationship due to misclassification effects $(7,8)$. Another study showed that increased diet quality (measured using the Alternate Mediterranean Diet score) was associated with a lower ectopic fat gain in the following 6 years (26). Similarly, increase in plant-centered diet quality (assessed using the APDQS) was associated with less weight gain from adolescence to early adulthood (12).

Some methodological uniqueness of the APDQS merits further discussion. Previous studies were mostly based on self-identified vegetarians (dichotomous form) or rare eaters of meats rather than on total diet quality (4). However, this simplified definition of a plant-centered diet overlooks the remaining parts of an individual's diet and is likely to result in misclassification bias. The way that the APDQS is structured allows flexible choice by providing a wide range of options and emphasizing variety (46 groups). Diversified food groups were equally weighted in the APDQS, with a maximum of four points; therefore, points need to come from many food groups to achieve a higher score. In contrast, the HEI-2015 and the AHEI-2010 each rely on a small number of food groups $(\leq 13)$ and are limited by the fact that individuals can get many points from single food groups and would not lose many points for eating less healthy foods.

The health benefits of plant-centered diets may relate to food synergy, defined as the concerted action of nutrients and bioactive compounds in individual foods, in meals and over time (27). This theory states that it is challenging to disentangle one factor from various food constituents to understand the protective effects of plant-centered diets. Nevertheless, constituents may be conceptually important. Fruits, vegetables, whole grains, and nuts contain abundant and varied amounts of antioxidants (vitamins A, C, and E and $\beta$-carotene), dietary fibers, and polyphenols $(28,29)$. Antioxidants may protect against progressive pancreatic $\beta$-cell impairment and endothelial dysfunction, which may plausibly reduce diabetes risk $(30,31)$. Polyphenols have antioxidant, anti-inflammatory, and antiplatelet properties and also lead to improvements in endothelial function, insulin sensitivity, and blood pressure (32). Dietary fiber in plant foods (soluble and insoluble) contributes to increased postprandial satiety, suppressed subsequent hunger, and decreased energy intake (33). Soluble fiber may also attenuate the rapid rise in the postprandial glucose response after a meal (34). All of these factors may partially explain the beneficial effect of a plant-centered diet on diabetes and weight control.

Strengths of the current study include its prospective design with high participant retention over a long follow-up, standardized and repeated assessment of covariates, detailed assessment of diet through the diet history interviewer, and collecting objective information on case subjects with incident type 2 diabetes and anthropometric measurements. Change analysis can strengthen temporality assertions and eliminate within-person confounding. Although change analysis is more challenging to interpret compared with baseline analysis, in particular because of the regression to the mean phenomenon, explicit consideration of baseline levels tends to resolve this difficulty. Our study also has several limitations. Observational studies cannot establish causal relationships, partly because of unmeasured or residual confounding. However, we adjusted for potential confounders for the association between diet and diabetes and body size. Selection bias may have occurred, but some participants had to be excluded in order to analyze the 20-year change in diet quality and preserve the quality of the data. The potential fluctuations in dietary pattern over time merit further investigation. In the current study, change in diet quality was estimated from two points in examination years ( $\mathrm{YO}$ and $\mathrm{Y} 20$ ) and thus may not have fully captured change in dietary pattern at the middle point during the study follow-up. Thus, potential misclassification may attenuate the association toward the null. However, prior CARDIA analysis showed that CARDIA participants had stable dietary patterns over 20 years, with tracking coefficients of 0.57 for Whites and 0.43 for Blacks (between Y0 and Y20 APDQS) (35). Moreover, analysis of short-term change in APDQS corroborated the main findings of the association between 20-year change in APDQS and diabetes and change in 
body size. We caution that differences in culture, race/ethnicity, and period of life can influence participant behaviors and may limit the generalizability of the results.

In conclusion, these findings provide evidence that young adults who consumed a plant-centered, high-quality diet and increased their diet quality over time had a lower risk of type 2 diabetes and smaller increases in body size as they aged through middle age. Our study suggests that eating a plant-centered diet may reduce early risk of type 2 diabetes and obesity.

Funding. The CARDIA study is supported by National Heart, Lung, and Blood Institute contracts HHSN268201800003I, HHSN268201800004I, HHSN268201800005I, HHSN268201800006I, and HHSN2682018000007I. Y.C. is supported by a Graduate and Professional Research Grant from the Healthy Food, Healthy Lives Institute and the MnDRIVE Global Food Ventures Professional Development Program, University of Minnesota Twin Cities.

The sponsor, the National Heart, Lung, and Blood Institute, has a representative on the Steering Committee of CARDIA and participated in study design, data collection, and scientific review of this article. The sponsor had no role in data analysis, data interpretation, or writing of this report.

Duality of Interest. D.D.G. is a paid member of the Nutrition Advisory Council for the California Prune Board. L.M.S received a grant ending in February 2020 with Dairy Management Inc. about dairy products. D.R.J. has been a paid consultant to the California Walnut Commission. No other potential conflicts of interest relevant to this article were reported.

Author Contributions. Y.C. and D.R.J. conceived and designed the study, conducted data analyses, and obtained funding for this study. Y.C. drafted the report. All authors contributed to data interpretation and critical review of the report and approved the final version of the manuscript. D.R.J. is the guarantor of this work and, as such, had full access to all of the data in the study and takes responsibility for the integrity of the data and the accuracy of the data analysis.

Prior Presentation. Parts of this study were presented in poster form at the American Society for Nutrition's Emerging Leaders in Nutrition Science Finalist Competition and as an oral session at Nutrition 2019, the flagship meeting of the American Society for Nutrition, Baltimore, MD, 8-11 June 2019 (Y.C.). Parts of this study were also presented as an oral session at the 78th Scientific Sessions of the American Diabetes Association, Orlando, FL, 22-26 June 2018 (D.R.J.).

\section{References}

1. Kodama S, Horikawa C, Fujihara K, et al. Quantitative relationship between body weight gain in adulthood and incident type 2 diabetes: a meta-analysis. Obes Rev 2014;15:202-214 2. Chen $C, Y e Y$, Zhang Y, Pan XF, Pan A. Weight change across adulthood in relation to all cause and cause specific mortality: prospective cohort study. BMJ 2019;367:15584
3. Willett W, Rockström J, Loken B, et al. Food in the Anthropocene: the EAT-Lancet Commission on healthy diets from sustainable food systems. Lancet 2019;393:447-492

4. Qian F, Liu G, Hu FB, Bhupathiraju SN, Sun Q. Association between plant-based dietary patterns and risk of type 2 diabetes: a systematic review and meta-analysis. JAMA Intern Med 2019;179:1335-1344

5. Rosell M, Appleby P, Spencer E, Key T. Weight gain over 5 years in 21,966 meat-eating, fisheating, vegetarian, and vegan men and women in EPIC-Oxford. Int J Obes 2006;30:1389-1396

6. Ley SH, Pan A, LiY, et al. Changes in overall diet quality and subsequent type 2 diabetes risk: three U.S. prospective cohorts. Diabetes Care 2016;39:2011-2018

7. Fung TT, Pan A, Hou T, et al. Long-term change in diet quality is associated with body weight change in men and women. J Nutr 2015;145: 1850-1856

8. Satija A, Malik V, Rimm EB, Sacks F, Willett W, $\mathrm{Hu} F \mathrm{FB}$. Changes in intake of plant-based diets and weight change: results from 3 prospective cohort studies. Am J Clin Nutr 2019;110:574-582

9. U.S. Department of Health and Human Services and U.S. Department of Agriculture. 2015-2020 Dietary Guidelines for Americans, 8th edition, 2015. Accessed 1 November 2019. Available from https://health.gov/dietaryguidelines/2015/ guidelines/

10. Lockheart MS, Steffen LM, Rebnord HM et al. Dietary patterns, food groups and myocardial infarction: a case-control study. Br J Nutr 2007;98:380-387

11. Hirahatake KM, Jacobs DR Jr, Shikany JM, Jiang L, Wong ND, Odegaard AO. Cumulative average dietary pattern scores in young adulthood and risk of incident type 2 diabetes: the CARDIA study. Diabetologia 2019;62:2233-2244 12. Hu T, Jacobs DR Jr, Larson NI, Cutler GJ, Laska $M N$, Neumark-Sztainer D. Higher diet quality in adolescence and dietary improvements are related to less weight gain during the transition from adolescence to adulthood. J Pediatr 2016; 178:188-193.e3

13. Friedman GD, Cutter GR, Donahue RP, et al. CARDIA: study design, recruitment, and some characteristics of the examined subjects. J Clin Epidemiol 1988;41:1105-1116

14. McDonald A, Van Horn L, Slattery M, et al. The CARDIA dietary history: development, implementation, and evaluation. J Am Diet Assoc 1991:91:1104-1112

15. Liu K, Slattery M, Jacobs D Jr., et al. A study of the reliability and comparative validity of the cardia dietary history. Ethn Dis 1994;4:15-27 16. Jacobs DR Jr., Hahn LP, Haskell WL, Pirie P, Sidney $\mathrm{S}$. Validity and reliability of short physical activity history: CARDIA and the Minnesota Heart Health Program. J Cardiopulm Rehabil 1989;9: 448-459

17. Hertzmark E, Pazaris $M$, Spiegelman D. The SAS mediate macro, 2018. Accessed 26 March 2020. Available from https://www.hsph.harvard .edu/donna-spiegelman/software/mediate/

18. Durrleman S, Simon R. Flexible regression models with cubic splines. Stat Med 1989;8:551561

19. Xu Z, Steffen LM, Selvin E, Rebholz CM. Diet quality, change in diet quality and risk of incident CVD and diabetes. Public Health Nutr 2020;23:329-338
20. Gijsbers L, Ding EL, Malik VS, de Goede J, Geleijnse JM, Soedamah-Muthu SS. Consumption of dairy foods and diabetes incidence: a dose-response meta-analysis of observational studies. Am J Clin Nutr 2016;103: 1111-1124

21. Djoussé L, Khawaja OA, Gaziano JM. Egg consumption and risk of type 2 diabetes: a metaanalysis of prospective studies. Am J Clin Nutr 2016;103:474-480

22. Namazi N, Brett NR, Bellissimo N, Larijani B, Heshmati J, Azadbakht L. The association between types of seafood intake and the risk of type 2 diabetes: a systematic review and metaanalysis of prospective cohort studies. Health Promot Perspect 2019;9:164-173

23. Yang $X, L i$, Wang $C$, et al. Meat and fish intake and type 2 diabetes: dose-response metaanalysis of prospective cohort studies. Diabetes Metab. 14 April 2020 [Epub ahead of print]. DOI: 10.1016/j.diabet.2020.03.004

24. Tonstad S, Butler T, Yan R, Fraser GE. Type of vegetarian diet, body weight, and prevalence of type 2 diabetes. Diabetes Care 2009;32:791796

25. Huang RY, Huang CC, Hu FB, Chavarro JE. Vegetarian diets and weight reduction: a metaanalysis of randomized controlled trials. J Gen Intern Med 2016;31:109-116

26. Hennein R, Liu C, McKeown NM, et al. Increased diet quality is associated with long-term reduction of abdominal and pericardial fat. Obesity (Silver Spring) 2019;27:670-677

27. Jacobs DR Jr, Steffen LM. Nutrients, foods, and dietary patterns as exposures in research: a framework for food synergy. Am J Clin Nutr 2003;78(Suppl.):508S-513S

28. Velioglu YS, Mazza G, Gao L, Oomah BD. Antioxidant activity and total phenolics in selected fruits, vegetables, and grain products. $J$ Agric Food Chem 1998;46:4113-4117

29. Ros E, Hu FB. Consumption of plant seeds and cardiovascular health: epidemiological and clinical trial evidence. Circulation 2013;128:553565

30. Varadharaj S, Kelly OJ, Khayat RN, Kumar PS, Ahmed N, Zweier JL. Role of dietary antioxidants in the preservation of vascular function and the modulation of health and disease. Front Cardiovasc Med 2017;4:64

31. Karunakaran U, Park KG. A systematic review of oxidative stress and safety of antioxidants in diabetes: focus on islets and their defense. Diabetes Metab J 2013;37:106-112

32. Pandey KB, Rizvi SI. Plant polyphenols as dietary antioxidants in human health and disease. Oxid Med Cell Longev 2009;2:270278

33. Howarth NC, Saltzman E, Roberts SB. Dietary fiber and weight regulation. Nutr Rev 2001;59: 129-139

34. Braaten JT, Scott FW, Wood PJ, et al. High beta-glucan oat bran and oat gum reduce postprandial blood glucose and insulin in subjects with and without type 2 diabetes. Diabet Med 1994;11:312-318

35. Sijtsma FP, Meyer KA, Steffen LM, et al. Longitudinal trends in diet and effects of sex, race, and education on dietary quality score change: the Coronary Artery Risk Development in Young Adults study. Am J Clin Nutr 2012;95: 580-586 